du 31 mars 1954 (J.O. 23 avril 1954) et Norme française. AFNORV 04-214, homologuée le 31 mars 1954 (J.O. 23 avril 1954).

[2] J. Pien. L'avenir de la méthode Gerber. C.R. du Congrès Section III. Question 4.

[3] G. Posthumus. Netherl. Milk and Dairy Journ, V. (1951), 10.

[4] J. Pien. Conditions à remplir par les pipettes à lait pour butyrométrie. C. R. du Congrès, Section III. Question 4.

\title{
LE LAIT DANS LE MONDE
}

\author{
PAR \\ G. GENIN \\ Ingénieur E. P. C.
}

\section{ANGLETERRE}

\section{La stérilisation à très haute température du lait}

$\mathrm{Au}$ Congrès de la Society of Dairy Technology, qui s'est tenu à Bournville, le Dr L.F.L. ClegG du National Institue for Research in Dairying a présenté une communication sur les tendances futures dans l'évolution des procédés de stérilisation du lait.

Une de ces tendances se manifeste par l'adoption de températures de stérilisation de plus en plus élevées, la durée du traitement étant par contre sensiblement réduite. Les raisons de cette tendance sont dues au fait que certains microorganismes, qui sont la cause de l'altération du lait pasteurisé ou du lait stérilisé, ne sont pas détruits par les méthodes actuelles de traitement.

On oublie en effet trop souvent de se rappeler que le lait stérilisé n'est pas en général rigoureusement stérile et qu'il peut donc s'altérer s'il est conservé dans des conditions défectueuses, surtout en été. Ce n'est que par un traitement à plus haute température qu'on parvient à détruire ces germes, mais un tel traitement a pour conséquence généralement d'altérer le goût du lait. On recherche done des procédés industriels permettant d'atteindre ces hautes températures, se façon à détruire les spores des bactéries, la durée de traitement du lait ne dépassant pas 1 à 2 secondes.

\section{Nouveau dispositif de distribution du lait}

Un nouveau système de distribution du lait a été récemment utilisé avec succès au milk-bar du National Milk Publicity Council, faisant partie de l'Espoxition britannique intitulée "La Maison idéale ". Pour distribuer le lait à 10 points de vente différents du Bar, il avait été prévu deux réservoirs cylindriques horizontaux, d'une capacité de près de 500 litres, exécutés en acier stainless et recevant de l'air comprimé sous une pression d'environ 200 grammes 
par $\mathrm{cm}^{2}$. Cette pression était suffisante pour obliger le lait à gagner les points de distribution, le transport du lait s'effectuant par l'intermédiaire de tuyaux transparents en chlorure de polyvinyle.

\section{La conservation des produits alimentaires par traitement au moyen de radiations ionisantes}

La British Food Manufacturing Industries Research Association a décidé de collaborer à un nouveau plan d'études établi par les Services scientifiques du Gouvernement en vue d'examiner l'emploi des radiations ionisantes pour la conservation des aliments.

Le Dr F.H. BANField, Directeur de cette Association, a annoncé qu'un groupe de recherches va être établi à Cambridge afin de procéder aux études nécessaires dont les résultats seront communiqués aux industries intéressées. L'idée de l'utilisation des radiations ionisantes découle du fait que ces radiations détruisent certains insectes, ainsi que des micro-organismes et on a donc pensé à assurer la stérilisation des produits alimentaires par un traitement de ce genre. Il est nécessaire cependant d'établir si un tel traitement ne modifie pas chimiquement la composition des produits alimentaires, en particulier des graisses et des protéines, il faudra ensuite procéder à des études biologiques sur la consommation des aliments ayant subi un traitement d'irradiation.

\section{Nouvelles pellicules pour l'emballage des produits alimentaires}

La Goodyear tire and Rubber Co Ltd a présenté deux nouvelles qualités de Pliofilm, pellicule plastique susceptible d'être utilisée pour l'emballage des produits alimentaires, en particulier des produits vendus dans les magasins à self-service.

L'un de ces produits, dénommé FM-1, est plus particulièrement destiné à l'emballage de la viande, de la volaille et du poisson, en particulier des produits qui doivent être conservés à froid. Les qualités élastiques de cette pellicule lui permettent d'épouser les formes les plus diverses des produits à emballer. La qualité 75-BF se caractérise surtout par son imperméabilité, ce qui lui permet de conserver la saveur des produits emballés, cette qualité convient plus particulièrement à l'emballage du beurre, du fromag e, etc...

\section{EIRE}

\section{Une nouvelle laiterie moderne?}

Le Ministre de l'agriculture a inauguré officiellement, il y a quelques mois, une nouvelle installation de pasteurisation du lait, située dans un faubourg de Cork. Cette installation est sous le 
contrôle de la Société coopérative des producteurs de lait de la région de Cork et dès à présent elle peut traiter près de 35.000 litres de lait par jour, mais sa capacité de traitement pourra être facilement portée à 45.000 litres.

L'établissement de cette installation pour la pasteurisation du lait est la conséquence des recommandations faites par le Ministre de la Santé publique du Gouvernement de l'Eire qui avait recueilli les doléances de consommateurs se plaignant de ce que le lait livré en été était très rapidement sur et devenait inconsommable.

\section{Consommation"de fromagel}

On a enregistré depuis quelques années un développement sensible de la consommation de fromage qui a pratiquement doublé. On donne deux raisons principales à cette situation: l'une est l'augmentation du prix de la viande et la seconde le fait que les consommateurs irlandais se sont rendus compte de la valeur du fromage comme aliment.

Le peuple irlandais n'avait jamais été un important consommateur de fromage et aujourd'hui encore, malgré que cette consommation ait doublé par rapport aux années d'avant-guerre, elle est encore de seulement 550 grammes en moyenne par an et par habitant, alors que dans certains pays de l'Europe continentale elle dépasse 9 kilogrammes. La consommation du pays varie entre 2.000 et 2.500 tonnes, mais on pense qu'elle va encore se développer et près de $95 \%$ du fromage consommé sont produits sur place.

\section{Production laitière}

En dépit des réclamations de la part des producteurs que la production laitière n'est pas payante, la production du lait en 1956 a été beaucoup plus importante que dans n'importe quelle autre année précédente. Dans la république irlandaise, l'augmentation de la production a été plus de 2 millions de litres et dans l'Irlande du nord elle a atteint 500.000 litres.

Cette importante production a laissé également un excédent utilisable pour la production du beurre qui a atteint en 1956: $850.000 \mathrm{cwt}$, il en est résulté des possibilités intéressantes d'exportation qui, chose curieuse, n'ont pas été envoyées dans le RoyaumeUni, mais ont trouvé des acheteurs plus lointains.

\section{ALLEMAGNE}

\section{Nouvelle qualité de Polythène stérilisable}

Le Polythène a déjà été utilisé dans l'emballage de nombreux produits alimentaires, mais ce produit présente un inconvénient 
sérieux, c'est de ne pouvoir supporter les températures nécessaires pour la stérilisation de produits. On est parvenu à mettre au point en Allemagne la fabrication d'un nouveau type de Polythène susceptible de résister à des températures de l'ordre de $110^{\circ}$, sans altération ou déformation.

Ce Polythène est fabriqué par polymérisation de l'éthylène à une pression beaucoup plus faible que le Polythène ordinaire, il a une plus forte densité, et il peut supporter des stérilisations répétées. Il est plus rigide que le Polythène normal et a déjà servi à la fabrication de divers récipients stérélisables.

\section{AUTRICHE}

\section{Le transport du lait par pipe-line}

La plus grande partie du lait autrichien est produite pendant les mois d'été par des troupeaux de vaches qui se trouvent sur les alpages et le problème s'est toujours posé de transporter le lait produit chaque jour dans les établissements de traitement situés au fond des vallées. Ce transport a toujours représenté une opération onéreuse.

Il y a quelques années, l'Association coopérative laitière de Mittersil avait envisagé la construction d'une télé-benne pour relier les mâturages alpestres situés à une altitude de plus de 2.000 mètres aux laiteries situées dans les vallées, dans la province de Salzbourg. Mais ce projet avait dû être abandonné, comme se révélant trop onéreux.

L'Association a alors adopté une solution plus simple et moins coûteuse : celle de la construction d'un pipe-line en matière plastique. On peut dire dès à présent que l'exploitation de ce pipe-line s'est révélée très économique. La longueur de la tuyauterie installée est de plus de 2 kilomètres et le lait parcourt cette tuyauterie en 21 minutes. L'expérience a montré qu'au cours de ce transport, le lait ne subit absolument aucune altération.

\section{DANEMARK}

\section{Fourniture de lait à l'armée américaine d'occupation en Allemagne}

Les Coopératives laitières danoises ont signé un important contrat pour la fourniture de lait aux troupes américaines stationnées en Allemagne, portant sur une livraison de plus de 10 millions de litres. Il a été prévu en outre que $60 \%$ du lait ainsi livré seront emballés dans des emballages en carton et les américains ont fourni les capitaux nécessaires pour la construction d'une installation 
permettant de fabriquer 40.000 emballages à l'heure, ainsi que les installations de remplissage. Le lait est livré en Allemagne par un train spécial qui chaque jour arrive du Danemark.

\section{Industrie de la crème glacée}

Il semble que ce soit l'industrie américaine qui ait donné une impulsion définitive au développement de la fabrication de la crème glacée au Danemark. C'est ainsi par exemple que la plus importante fabrique de crème glacée située à Copenhague, la firme Hellerepis, est la propriété de la famille JENSEN dont la formation a été faite aux Etats-Unis. D'autre part, le professeur R. MortenSEN qui, pendant de nombreuses années, avait dirigé l'Institut laitier de l'Iowa State College était retourné en 1926 au Danemark pour y poursuivre son enseignement.

La production de crème glacée danoise a été en 1954 de 3,7 millions de gallons soit 0,8 gallons par habitant. La crème danoise contient en moyenne 12, 5\% de graisse et cette graisse est obligatoirement de la graisse extraite du lait. La teneur en sucre s'élève en moyenne à $11 \%$. La gélatine est le plus utilisé des agents stabilisants, mais on emploie également des alginates, ainsi que certains dérivés cellulosiques.

\section{Formation de spécialistes laitiers}

C'est à Copenhague que seront formés les spécialistes chargés du développement de l'industrie laitière dans les pays sous-alimentés. C'est en effet d'accord entre le gouvernement danois et la F.A.O. que des spécialistes provenant du Cambodge, de Ceylan, de l'Inde, d'Indonésie, du Pakistan et de la Thailand, doivent recevoir au Danemark à la fois un enseignement théorique et un enseignement pratique au Collège royal d'agriculture et au Collège vérétinaire de Copenhague. Entre les périodes de cours, les étudiants passeront également un certain temps dans différentes exploitations laitières, qu'il s'agisse de fermes ou de laiteries coopératives.

\section{SUEDE}

\section{Les progrès de l'industrie laitière}

La Suède bénéficie aujourd'hui d'une industrie laitière parfaitement organisée. La totalité du lait vendu dans le pays, même dans les régions les plus isolées, est dirigée vers des laiteries collectives et jamais le lait n'est vendu directement parle fermier. Le prix de ce lait est établi d'après sa qualité hygiénique et sa teneur en graisse. En général les exploitations agricoles sont de peu 
d'importance, les fermes ne possédant que 6 à 8 vaches. Malgré cela, les procédés de traite mécaniques sont très développés.

Le climat permet d'éviter l'installation de postes de réfrigération importants et par exemple, on se contente de placer les barattes dans un courant d'eau froide. Le climat permet également de réduire les risques d'infection microbienne. En outre, les fermes sont très propres et les animaux restent à l'étable la plus grande partie de l'année.

On s'est surtout préoccupé de sélectionner les animaux de façon à produire un lait à haute teneur en graisse. C'est ainsi que la race frisienne noire, qui fournit un fort rendement en lait, mais un lait de faible teneur en graisse ne représente que $20 \%$ du troupeau suédois de vaches laitières, tandis que la race frisienne rouge, dont le rendement en lait est plus faible, mais qui fournit un lait plus riche en graisse représente $65 \%$ des animaux. C'est dans le nord du pays qu'on trouve principalement la race suédoise blanche.

\section{ITALIE}

\section{Production Iaitière}

D'après les statistiques récentes, il existait en Italie au 31 décembre 1954 : 9.032 .600 bêtes à cornes. C'est surtout dans le nord de l'Italie que se trouvent ces troupeaux, le sud du pays ne disposant que de très peu de vaches et consommant surtout du lait de brebis ou du lait de chèvres. Il existe en Italie plus de 8 millions de brebis et plus de 2 millions de chèvres; on trouve également dans certaines régions du pays des bufflesses.

La production totale laitière s'élève en moyenne à 1.890 millions de gallons. C'est surtout dans la région du Pô et dans les régions alpines que la production est la plus importante.

\section{Consommation de lait}

Environ le tiers du lait produit en Italie est consommé tel quel. La consommation annuelle par habitant s'établit à $\mathbf{5 0}$ kilogrammes, mais on observe de très grandes variations entre les différentes régions du pays. Dans le nord, elle est de 84 kilogrammes, dans le centre du pays de 31 , et dans le sud de 13,6 .

Dans le nord du pays, on enregistre une importante consommation de lait, surtout dans les régions rurales ou le produit est consommé d'une part par les producteurs et d'autre part par la famille des ouvriers agricoles, une partie des salaires étant payée sous la forme de produits laitiers. 


\section{Industrie du fromage}

La production de fromage est d'environ 350.000 tonnes, dont $75 \%$ sont produits dans le nord du pays. C'est le parmesan qui représente la production la plus importante.

Les exportations de fromage ont diminué régulièrement depuis quelques années, alors que les importations sont en croissance régulière depuis les mesures prises en vue de libérer les échanges commerciaux.

Actuellement, des études sont en cours afin de redresser cette situation, par une meilleure qualité des produits fabriqués en Italie et par un effort publicitaire destiné à favoriser les ventes de fromages italiens à l'étranger.

Les principaux types de fromages actuellement fabriqués dans le pays sont les suivants : l'Asiago fabriqué principalement en Vénétie, initialement en partant de lait de brebis mais aujourd'hui en partant principalement de lait de vache, le Bel Paese fabriqué en partant de lait de vache ainsi que le Caciocavallo dans la préparation duquel on utilise parfois une faible proportion de lait de brebis, le Fontina fabriqué dans la vallée d'Aoste par un pro cédé analogue à celui utilisé pour la fabrication du gruyère; le Grana dont il existe plusieurs variétés exportées parfois sous le nom de Parmesan, le Pecorino romano qui est le plus connu des fromages de brebis fabriqués en Italie, le Provolone, le Robiola et le Scarmorze qui est essentiellement un fromage de lait de bufflesse.

\section{HOLLANDE}

\section{Création d'usines hollandaises en Afrique du Nord}

La N.V. Sterovita Melkproducten d'Amsterdam a mis en service, il y a quelques mois, à Tripoli, une laitẹrie destinée à alimenter les forces américaines stationnées en Afrique du nord en lait et en produits laitiers; C'est la seconde installation que cette affaire crée en Afrique du nord. En mars 1955, elle avait mis en service à Casablanca une installation analogue.

\section{Production laitière}

Des indications publiées par le service néerlandais des statistiques montrent que pour le premier trimestre 1956 la quantité de lait fourni par les producteurs a dépassé largement celle livrée pendant la même période de 1955, la teneur en graisse étant également plus élevée.

La production de beurre, de fromage, de lait condensé de lait en poudre pour les premiers trimestres 1956 et 1955 est donnée dans le tableau suivant. 
Lait livré par les producteurs, en millions de kilogrammes.................

Teneur en graisse en $\% \ldots \ldots \ldots \ldots \ldots \ldots$

Production en tonnes:

\begin{tabular}{|c|c|c|}
\hline beurre $\ldots \ldots \ldots$. & 11.681 & 12.957 \\
\hline fromage $\ldots \ldots \ldots \ldots \ldots \ldots \ldots \ldots$ & 23.496 & 25.620 \\
\hline lait condensé $\ldots \ldots \ldots \ldots \ldots \ldots \ldots \ldots$ & 50.984 & 54.116 \\
\hline lait en poudre $\ldots \ldots \ldots \ldots \ldots \ldots \ldots$ & 4.700 & 7.572 \\
\hline
\end{tabular}

\section{L'expédition de poudre de lait à l'Inde et au Pakistan}

Si le Gouvernement donne son accord, l'industrie néerlandaise des produits laitiers se joindra aux Etats-Unis, à l'Australie et à la Nouvelle Zélande pour fournir à l'Inde et au Pakistan, pendant une période de trois années, de la poudre de lait écrémé.

Ce projet fait partie d'un plan établi par les experts de la F.A.O. afin de développer la consommation du lait dans l'Inde et au Pakistan. Il a été convenu que la Commission néerlandaise des produits laitiers fournira ce lait écrémé à un prix réduit.

\section{Production de fromage}

Les statistiques définitives concernant la production de fromage en 1955 font apparaître une production record de 380.672 .600 pounds, en augmentation de $6 \%$ sur celle de 1954.

L'augmentation de la production intéresse toutes les variétés de fromage. La production des fromageries s'est élevée à 347.012.600 pounds, en augmentation de $6,1 \%$ sur celle de 1954 , et la production fermière a atteint 33.660 .000 pounds, en augmentation de $1,7 \%$. L'augmentation a surtout été importante pour les types suivants : Edam, Leyde et fromage de Frise. La production du Gouda est simplement en augmentation de 5,4\%. Il est vrai que c'est la production de ce fromage qui représente la plus forte proportion du total.

\section{YOUGOSLAVIE}

\section{Centre de formation pour spécialistes de l'industrie laitière}

La Food and Agriculture Organisation (F.A.O.), en collaboration avec le Gouvernement yougoslave, a constitué à Zagreb un nouveau Centre de formation des spécialistes de l'industrie laitière où les techniciens des pays du sud-est de l'Europe et de l'est de la méditerranée recevront une formation d'une durée de quatre semaines.

Les participants auront l'occasion d'étudier les derniers progrès réalisés dans l'industrie laitière et ils auront à charge de favoriser 
la production et la consommation du lait tel quel et des produits laitiers dans une région de l'Europe où cette consommation est encore très réduite.

L'enseignement portera sur les soins à donner aux animaux, la sélection des vaches laitières, la production du lait, sa transformation et sa distribution. On insistera en particulier sur les problèmes que pose l'aspect hygiénique de la distribution du lait.

\section{POLOGNE}

\section{Récentes recherches sur la fabrication du fromage}

Des recherches ont été entreprises en Pologne sur l'emploi des rayons $\mathrm{X}$ pour accélérer la maturation du fromage d'Edam. On a constaté qu'un émetteur de rayons ultraviolets du type Philipps U.F. de 30 watts installé dans un magasin de $55 \mathrm{~m}^{2}$ de surface détruit, après 4 heures d'irradiation, environ $80 \%$ des bactéries présentes. Dans des magasins contenant des fromages et dont l'atmosphère est soumise à l'irradiation, les fromages qui reçoivent directement les rayons ultraviolets ne se recouvrent pas de moisissures et sur les autres, la formation de moisissures est plus lente que dans les chambres non irradiées. Enfin, les fromages irradiés pendant une période de 14 jours ont une saveur plus marquée, ce qui semble indiquer que l'irradiation par les rayons ultra-violets accélère la maturation.

D'autres études ont également été faites sur la pasteurisation du lait employé dans la fabrication du fromage d'Edam. On a étudié trois modes différents de pasteurisation : par chauffage à $67^{\circ}$ pendant 3 minutes, par chauffage à $70-78^{\circ} \mathrm{C}$. pendant 30 à 48 secondes et par chauffage à $64.70^{\circ} \mathrm{C}$, pendant 30 à 48 secondes. C'est ce dernier procédé qui a permis d'obtenir les meilleurs résultats.

\section{ETATS-UNIS}

\section{Distribution des surplus agricoles américains}

La distribution des surplus agricoles américains se poursuit, en dépit des protestations des pays dont le commerce est handicapé par la vente sur le marché de produits à des prix non commerciaux. Une banque, l'Export-Surplus Bank des Etats-Unis, accorde aux acheteurs étrangers des emprunts à court terme pour leur permettre l'achat de ces surplus.

Une des dernières décisions prises par l'organisation chargée de l'écoulement de ces produits est la mise en vente de beurre en poudre dont la fabrication a été entreprise par une firme privée avec l'aide du Gouvernement. 100.000 pounds de ce produit dé- 
nommé Beacreme ont été expédiés en Suède, en Finlande et en Suisse. Le Beacreme est une combinaison de beurre et de poudre de lait dégraissé et il est surtout destiné à des fabrications industrielles, par exemple la préparation de pâtisserie. Il renferme $72 \%$ de graisse.

\section{L'emploi des radiations pour la conservation des denrées périssables}

En 1948, il existait trois Laboratoires étudiant l'emploi des radiations pour la conservation des denrées périssables, en 1954 ce nombre était de vingt et en 1955 il atteignait cinquante. En outre vingt-quatre sociétés ont commencé à étudier l'emploi des réacteurs nucléaires dans le traitement des produits alimentaires.

Il est encore trop tôt pour établir les résultats de ces recherches, mais il semble qu'après un traitement par les radiations, beaucoup de produits alimentaires peuvent être conservés à la température ordinaire ou à une température assez fraîche, sans exiger de véritable congélation. On a également constaté que l'influence des radiations est négligeable sur les graisses et sur les protéines, une partie des vitamines est détruite, mais pas plus qu'après une stérilisation par la chaleur.

\section{L'emploi du polyéthylène pour l'expédition de la crème fraîche}

On a commencé dans certaines régions des Etats-Unis à utiliser des sacs en polyéthylène pour l'expédition de la crème entre les fermes productrices et les usines laitières. Ces sacs sont directement remplis de crème à la sortie de l'écrémeuse et sont fermés par une bande élastique. Chaque sac contient environ cinq kilogrammes de crème, le produit est maintenu dans un réfrigérant jusqu'au moment de l'envoi et le transport s'effectue dans des boîtes en carton ondulé qui assurent un isolement thermique suffisant pendant la durée du transport.

Ce mode d'emballáge permet d'économiser de la place et du poids, tout en assurant une parfaite conservation de la qualité du produit.

\section{Nouvel agent sucrant}

Ce produit, dénommé Cylan a été mis en vente depuis déjà quelque temps et il est constitué essentiellement de cyclamate de calcium. Il est destiné plus particulièrement à la préparation d'aliments pour régimes, et en particulier d'aliments pour diabétiques ou pour personnes effectuant une cure d'amaigrissement. Il a l'avantage de ne laisser aucun goût dans la bouche et il peut 
subir l'action de la chaleur, sans perte de son pouvoir sucrant. Il peut en particulier être utilisé dans la préparation de boissons à base de lait devant présenter un goût sucré.

\section{Développement de l'emballage du lait en carton}

On estime qu'à l'heure actuelle, plus de $54 \%$ du lait vendu tel quel pour la consommation directe, est maintenant livré dans des emballages en carton paraffiné. La Division Pure Pak de l'Ex-Cell-O Corporation de Detroit estime par exemple qu'en 1955 il a été fabriqué près de 12 millions d'emballages carton contre seulement 2 millions il y a dix ans. La même société annonce que, alors qu'en 1945 les récipients en verre étaient utilisés pour $90 \%$ des livraisons de lait aux consommateurs, on prévoit qu'en 1960, ce pourcentage sera inférieur à $25 \%$.

\section{La production d'air exempt de bactéries}

Le Centre de recherches bactériologiques de l'University of California a récemment mis au point un procédé de purification de l'air destiné à l'aération des laboratoires ce bactériologie. Ce procédé permet en effet d'éliminer $99 \%$ des organismes microbiens existant dans l'air et il pourrait par exemple être utilisé pour la purification de l'air circulant dans les salles de maturation du fromage.

La purification de l'air est assurée par des lampes germicides qui agissent sur l'air circulant dans un diffuseur construit par la Pyle-National Co de Chicago. Dans ce diffuseur l'air ne circule qu'à la vitesse de seulement 90 mètres à la minute, ce qui contribue à provoquer une destruction presque totale des bactéries présentes.

\section{BIBLIOGRAPHIE ANALYTIQUE}

\section{LES LIVRES}

Dehove (Raymond). - La réglementation des produits alimentaires et non alimentaires, et la répression des fraudes. 1 volume relié, $16 \times 24$, de 584 pages, troisième édition. Editeur : Commerce-Editions, 2, rue des Petits-Pères, Paris $\left(2^{\mathrm{e}}\right) .1957$. Prix : 2.000 franes; franco : 2.150 francs.

Présenté par $M M$. Chauvet, Inspecteur Général, Chef du Service de la Répression des Fraudes, Lemoigne, Membre de l'Institut, Chef de Service à l'Institut Pasteur, et Manaut, Président de la Fédération Nationale de l'Industrie de l'Alimentation, cet ouvrage, dont les deux premières éditions ont été présentées à nos lecteurs, est à jour des diverses réglementations. 